\title{
Impact of Multiple Primaries and Partial CSI on Transmit Antenna Selection for Interference-Outage Constrained Underlay CR
}

\author{
Rimalapudi Sarvendranath $^{\circledR}$, Student Member, IEEE, and Neelesh B. Mehta ${ }^{\circledR}$, Fellow, IEEE
}

\begin{abstract}
Transmit antenna selection is a low-complexity multiple-antenna technique that exploits spatial diversity using only one radio frequency chain. We investigate it for an underlay cognitive radio system that operates in the presence of multiple primary receivers and is subject to a constraint on the interference outage it causes at any of the primary receivers. The selection is based on a practically motivated and general partial channel state information (CSI) model in which the secondary transmitter (STx) only knows the channel power gains to a subset of the primary receivers. We derive a novel and general antenna selection rule that provably minimizes the symbol error probability (SEP) of the secondary system. We also derive insightful analytical expressions for its average SEP and interference-outage probability. These apply to a general class of channel fading models and any number of transmit and receive antennas, and include the special cases in which the STx knows channel power gains of all or none of the primary receivers. Our numerical results bring out a new insensitivity of the average SEP of the optimal rule to the interference power threshold when the CSI available is partial.
\end{abstract}

Index Terms-Cognitive radio, underlay, antenna selection, interference outage, multiple primaries, partial CSI.

\section{INTRODUCTION}

$\mathbf{C}$ OGNITIVE radio $(\mathrm{CR})$ is a promising technology that addresses the shortage of radio spectrum. Owing to its promise, it has been incorporated in IEEE standards such as 802.11af and 802.22 [1]. In CR, users are classified into two categories, namely, primary users (PUs), who are licensed users of the spectrum, and secondary users (SUs), who are lower priority users of the same spectrum [2]. We focus on the underlay paradigm of $\mathrm{CR}$, which improves frequency reuse by allowing a SU to transmit concurrently in the same spectrum as the PU. However, the SU is subject to a constraint on the interference its transmissions cause to the primary receivers (PRxs) [2].

Manuscript received May 3, 2018; revised August 12, 2018 and November 25, 2018; accepted December 4, 2018. Date of publication December 21, 2018; date of current version February 11, 2019. This work was supported in part by the Kaikini Ph.D. Scholarship in Engineering and the DST-Swarnajayanti Fellowship award DST/SJF/ETA-01/2014-15. The associate editor coordinating the review of this paper and approving it for publication was I. Guvenc. (Corresponding author: Rimalapudi Sarvendranath.)

The authors are with the Department of Electrical Communication Engineering, Indian Institute of Science, Bengaluru 560012, India (e-mail: sarvendranath@gmail.com; nbmehta@iisc.ac.in).

Digital Object Identifier 10.1109/TWC.2018.2886889
Different interference constraints have been considered in the literature to protect the PRxs from excessive interference. These include the peak-interference constraint [3]-[7], which limits the instantaneous interference power at the PRxs, the interference-outage constraint [8]-[10], which limits the fraction of time for which instantaneous interference can exceed a interference power threshold at the PRx, and the averageinterference constraint [11]-[13], which limits the fadingaveraged interference power at the PRx. However, these limit the secondary performance.

Multiple antennas at the SUs can ameliorate this performance degradation. For example, transmit beamforming is considered in [14], and multiple input multiple output (MIMO) antenna techniques are considered in [15]. However, these require an expensive and power-hungry radio frequency (RF) chain per antenna, which consists of digitalto-analog converters, filters, mixers, and an amplifier. Transmit antenna selection (TAS) is a technique that addresses this challenge [16]. In it, the transmitter uses a single RF chain, which is dynamically switched to one of the antennas depending on the channel conditions. It is employed in wireless standards such as IEEE 802.11n and Long Term Evolution (LTE) [17], [18].

In underlay CR, TAS reduces the symbol error probability (SEP) [10]-[12] and increases the ergodic capacity [6], [7] compared to a single antenna system that uses the same number of RF chains. The choice of the antenna depends not only on the channel gain between the secondary transmitter (STx) and secondary receiver (SRx), but also on the channel gains between the STx and the PRxs and the interference constraint itself [6], [10]. In the following, we summarize the TAS rules studied in the literature for underlay $\mathrm{CR}$.

For Single PRx: For an STx that transmits with fixed power, the antenna with the highest STx to SRx (STxSRx) channel power gain among the antennas that satisfy the peak-interference constraint is selected in [6]. Instead, in [7], the STx adapts its transmit power and selects the antenna with the highest ratio of the STx-SRx and the STx to PRx (STx-PRx) channel power gains. SEP-optimal TAS rules are developed in [11] and [12] for an STx that is subject to the average-interference constraint, and in [10] for 
an interference-outage constrained STx. A difference selection (DS) rule that selects the antenna with the maximum weighted difference of the STx-SRx and STx-PRx channel power gains is proposed in [13], and a minimum interference (MI) rule that selects the antenna with the smallest STx-PRx channel power gain is proposed in [19].

For Multiple PRxs: For an STx that can adapt its transmit power and is required to satisfy the peak-interference constraint at each of the PRxs, the antenna with the highest STx-SRx channel power gain is selected in [3] and [4]. A low complexity iterative algorithm for jointly determining the antenna subset and beamforming vector is proposed in [5].

\section{A. Focus and Contributions}

In this paper, we characterize how the presence of multiple PRxs, availability of channel state information (CSI) about the links to them, and the interference constraint together drive the optimal selection of the transmit antenna. We consider an STx that employs on-off power control. In the on state it transmits data with a fixed non-zero power and in the off state it transmits with zero power in order to not cause interference to the PRxs [6], [8], [11]. It is practically appealing because it enables the use of high-efficiency power amplifiers and also simplifies the design of the other components of the RF chain. We focus on the interference-outage constraint, which is a generalization of the widely studied peak-interference constraint [3]-[5]. Given its stochastic nature, STx need not perfectly know the instantaneous channel power gains from it to all the PRxs, which is unlike the peak-interference constraint. Moreover, it does not significantly affect primary systems that are designed to handle co-channel interference or whose traffic is tolerant to disruptions [10].

Several aspects of our model are novel. Firstly, the multiple PRxs scenario has been investigated much less in the literature. Secondly, we study a partial CSI model, in which the STx knows instantaneous channel power gains from it to only a subset of PRxs. This is motivated by the fact that while the STx can acquire CSI of its link to a PRx, this is more challenging with multiple PRxs. For example, consider a primary system that operates in the time division duplexing (TDD) mode. The STx listens to primary signals for a finite time period and exploits reciprocity in order to estimate the STx-PRx channel gains [20], [21]. In such a case, it can obtain CSI of only those PRxs that transmit during this period. The same issue arises even when the primary system operates in the frequency division duplexing (FDD) mode and a hidden power-feedback loop technique [22] is used to estimate the STx-PRx channel power gains. As the number of PRxs increases, acquiring CSI of the links to all the PRxs in a timely manner becomes difficult [23], [24]. Thirdly, we study the general case in which the channel gains from the STx to different PRxs are statistically non-identical. This models the scenario in which the PRxs are at different distances from the STx, and, thus, have different path-losses. Our model differs from [3]-[5], which assume the peak-interference constraint and statistically identical STx-PRx channel gains, all of which are known to the STx.
We make the following contributions:

- We develop an optimal TAS rule for on-off power control that minimizes the average SEP for a secondary system with partial CSI at the STx that is subject to a constraint on the interference-outage caused to any of the multiple PRxs. We present a novel and explicit mathematical characterization of it. It selects an antenna that minimizes a function that is a difference of two terms. The first term is an exponentially decaying function of instantaneous signal-to-noise-ratio (SNR) at the SRx. The second term is a product of three terms. The first is an indicator function that checks if the STx-PRx channel power gains that are known at the STx are below a threshold, the second is a constant that is a function of the channel statistics of the STx-PRx links that are not known at the STx, and the third is a penalization parameter $\lambda$ that depends on the parameters of the interference-outage constraint. The rule is optimal for any fading model with a continuous cumulative distribution function (CDF), which we shall refer to as a continuous fading model. This encompasses the Rayleigh, Rician, and Nakagami- $m$ models.

- We derive a general expression for the average SEP of the optimal rule and its interference-outage probability for any number of PRxs, for any number of antennas at the STx and SRx, and for many constellations. We also derive a tight and closed-form upper bound for the interferenceoutage probability, which simplifies the implementation of the optimal rule.

- We also study the following two extreme cases, which are by themselves novel and insightful: (i) Full CSI, in which the STx knows the channel power gains from it to all the PRxs; (ii) No CSI, in which the STx does not know the channel power gains to any of the PRxs.

- Our extensive simulation results show that the optimal rule reduces the average SEP markedly compared to the other TAS rules. An interesting insight we obtain is that with partial CSI the performance of the optimal rule is insensitive to the interference power threshold.

The multiple PRxs model considered is more general than the single PRx model considered in [6], [11]-[13], and [25]. Additionally, the partial CSI model and statistically nonidentical STx-PRx channel gain model are more general than the full CSI model assumed in [3]-[5]. Our paper differs in many ways compared to [10]. We consider the partial CSI model with multiple PRxs, which is more general that the full CSI model with single PRx considered in [10]. Secondly, the question of the different STx-PRx channel gains being statistically non-identical does not arise in [10]. Thirdly, the interference-outage constraint that we consider is novel compared to that in [10]. Consequently, the TAS rule that we present and prove to be optimal is different from that in [10]. This also entails more sophisticated average SEP and interference-outage probability analyses. We note that even the special case of no CSI that we study does not follow from [10].

\section{B. Outline and Notation}

Section II presents the system model and the problem statement. The optimal TAS rule and its interference-outage 


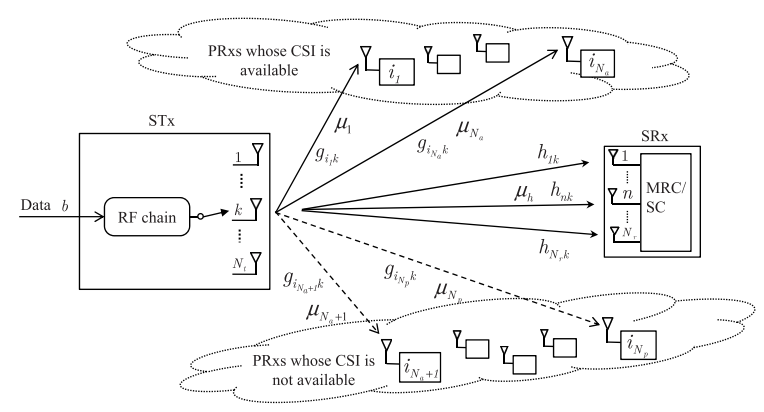

Fig. 1. System model that consists of an STx with $N_{t}$ transmit antennas and one RF chain. It transmits data to an SRx with $N_{r}$ antennas, which causes interference to $N_{p}$ PRxs.

probability are derived in Section III. Its average SEP is analyzed in Section IV. Performance benchmarking and results are presented in Section V. Our conclusions follow in Section VI. Mathematical derivations are relegated to the Appendix.

Notation: The absolute value of a complex number $x$ is denoted by $|x|$. The complement of a set $\mathcal{A}$ is denoted by $\mathcal{A}^{c}$. The probability of an event $A$ and the conditional probability of $A$ given $B$ are denoted by $\operatorname{Pr}(A)$ and $\operatorname{Pr}(A \mid B)$, respectively. $\mathbb{E}_{X}[\cdot]$ denotes expectation with respect to $X$. Further, $X \sim$ $\mathcal{C N}\left(\sigma^{2}\right)$ means that $X$ is a circular symmetric zero-mean complex Gaussian RV with variance $\sigma^{2}$. Scalar variables are written in normal font and vector variables in bold font. $I_{\{a\}}$ denotes the indicator function; it is 1 if $a$ is true and is 0 otherwise. The null set is denoted by $\varnothing$.

\section{System Model and Problem Statement}

The system model is shown in Fig. 1. It consists of an STx that transmits data to an SRx, which causes interference to $N_{p}$ PRxs. Each PRx is equipped with one antenna while the STx and SRx are equipped with $N_{t}$ and $N_{r}$ antennas, respectively. The STx is equipped with one RF chain, which dynamically connects it to one antenna depending on the channel gains. The SRx employs either maximal ratio combining (MRC) or selection combining (SC). The latter is practically motivated because it enables the use of fewer expensive RF chains at the SRx [3]. For $n \in\left\{1,2, \ldots, N_{r}\right\}$ and $k \in\left\{1,2, \ldots, N_{t}\right\}, h_{n k}$ denotes the instantaneous channel power gain from the $k^{\text {th }}$ antenna of the STx to the $n^{\text {th }}$ antenna of the SRx. The STx-SRx channel gains are statistically identical since the antennas at the STx are co-located and so are the antennas at the SRx. We also assume that these gains are mutually independent, as this makes the analysis tractable and is widely assumed in the related literature [6], [7], [11], [25]. Let $\mu_{h}$ denote the mean STx-SRx channel power gain and $\mathbf{H}=\left[h_{n k}\right]$ denote the STx-SRx channel power gain matrix. Using the simplified path-loss model [26, Ch. 2.6], $\mu_{h}$ in terms of the distance $d$ between the STx and SRx is equal to $(\Lambda /(4 \pi d))^{2}$, if $d \leq d_{0}$, and is $\left(\Lambda /\left(4 \pi d_{0}\right)\right)^{2}\left(d_{0} / d\right)^{\zeta}$, otherwise. Here, $\zeta$ is the path-loss exponent, $\Lambda$ is the signal wavelength, and $d_{0}$ is the breakpoint distance.

The instantaneous channel power gain from the $k^{\text {th }}$ antenna of the STx to the $i^{\text {th }}$ PRx is denoted by $g_{i k}$. The channel gains from the STx to a given PRx are assumed to be independent and identically distributed (i.i.d.), i.e., $g_{i 1}, \ldots, g_{i N_{t}}$ are i.i.d. for any given $i \in\left\{1,2, \ldots, N_{p}\right\}$. And, the channel power gains $g_{1 k}, \ldots, g_{N_{p} k}$ from antenna $k$ of the STx to the different PRxs are independent and not identically distributed (i.n.i.d.). Let $\mu_{i}=\mathbb{E}\left[g_{i k}\right]$. This models the scenario where the different PRxs are at different distances from the STx, as can be seen from the above path-loss model. All channel gains remain constant over a symbol duration, which is of the order of tens of microseconds or less.

\section{A. On-Off Power Control and Data Transmission}

The STx draws a data symbol $b$ from a constellation of $M$ symbols that are equally likely. It employs on-off power control, in which the STx transmits data either with power $P_{t}$ using one of the $N_{t}$ antennas or with 0 power in the on and off states, respectively [8], [11]. The zero transmit power option is equivalent to the STx transmitting from a virtual antenna 0 , with $h_{10}=\cdots=h_{N_{r} 0} \triangleq 0$ and $g_{10}=\cdots=g_{N_{p} 0} \triangleq 0$.

Let $s \in\left\{0,1, \ldots, N_{t}\right\}$ denote the antenna selected by the STx. At the $n^{\text {th }}$ antenna of the SRx, let $Z_{n}$ denote the interference from the PU transmissions and $R_{n}$ denote the signal received. Let the instantaneous interference at the $i^{\text {th }}$ PRx due to an STx transmission be $I_{i}$. Then,

$$
\begin{aligned}
R_{n} & =\sqrt{P_{t}} \sqrt{h_{n s}} e^{j \theta_{n s}} b+W_{n}+Z_{n}, \quad \text { for } 1 \leq n \leq N_{r}, \\
I_{i} & =\sqrt{P_{t}} \sqrt{g_{i s}} e^{j \varphi_{i s}} b, \quad \text { for } 1 \leq i \leq N_{p},
\end{aligned}
$$

where $\mathbb{E}\left[|b|^{2}\right]=1, \theta_{n s}$ and $\varphi_{i s}$ are the phases of the complex baseband STx-SRx and STx-PRx channel gains, respectively, and $W_{n}$ is circular symmetric complex additive white Gaussian noise. We assume $Z_{n}$ to be Gaussian. This is widely assumed in the literature to ensure tractability [8], [11]-[13]. It is justified by the central limit theorem when there are multiple primary transmitters (PTxs). It is valid even with one PTx and Rayleigh fading, when the PTx transmits a constant amplitude signal [8]. It is also valid for the model considered in [19] and [23], in which the SRx is located far from the PTx. Therefore, $W_{n}+Z_{n} \sim \mathcal{C N}\left(\sigma^{2}\right)$.

\section{B. CSI Model}

1) STx-SRx Channels: In order to perform TAS, we assume that the STx knows $\mathbf{H}$ [6], [7]. This can be obtained by exploiting reciprocity or using feedback. The STx does not need phase information of any STx-SRx channel. For coherent demodulation, the SRx knows the complex channel gains from the selected transmit antenna $s$ of the STx to its $N_{r}$ receive antennas, i.e., $h_{n s}$ and $\theta_{n s}$, for $n \in\left\{1,2, \ldots, N_{r}\right\}$. This can be achieved by inserting pilots along with the transmitted data [10], [11].

2) STx-PRx Channels: In the partial CSI model, the STx only knows the channel power gains from it to a subset $\mathcal{A}$ of $N_{a}$ PRxs. This arises because the STx can only estimate the channel gains of the PRxs that transmit in a finite time window. Let $\mathbf{G}_{\mathcal{A}}=\left[g_{i k}\right]$, for $i \in \mathcal{A}$ and $k \in\left\{1,2, \ldots, N_{t}\right\}$, denote the STx-PRx channel power gain sub-matrix. The following two are extreme cases of our general model: 
- Full CSI: Here, $\mathcal{A}=\left\{1,2, \ldots, N_{p}\right\}$ since the STx knows the channel power gains from it to all the PRxs.

- No CSI: Here, $\mathcal{A}=\varnothing$ since the STx does not know the channel power gains to any of the PRxs.

The STx has statistical information about all the STx-PRx links, e.g., their mean channel power gains, since it changes at a much slower rate than the instantaneous channel gains. No phase information of the STx-PRx channel gains is required at the STx.

\section{Interference-Outage Constraint and Problem Statement}

Interference-outage is an event in which the instantaneous interference at any of the PRxs exceeds an interference power threshold $\tau$. This is a generalization of the definition of interference-outage employed in [9], [10], and [23] for a system with one PRx. It also includes as a special case the constraint on the peak interference at each PRx, which is considered in [3]-[5].

From (2), the instantaneous interference power at the $i^{\text {th }}$ PRx is $P_{t} g_{i s}$. Then, an interference-outage occurs when $P_{t} g_{i s}>\tau$, for any $i \in\left\{1,2, \ldots, N_{p}\right\}$. We specify the interference-outage constraint as

$$
\operatorname{Pr}\left(\bigcup_{i=1}^{N_{p}}\left\{P_{t} g_{i s}>\tau\right\}\right) \leq O_{\max }
$$

where $O_{\max }$ is the maximum allowed interference-outage probability. It follows from (3) that $\operatorname{Pr}\left(P_{t} g_{1 s}>\tau\right) \leq$ $O_{\max }, \ldots, \operatorname{Pr}\left(P_{t} g_{N_{p} s}>\tau\right) \leq O_{\max }$. Therefore, it also constrains the interference-outage probability at each PRx regardless of its distance from the STx. Using the De Morgan's law, the constraint in (3) can be written as $\operatorname{Pr}\left(\cap_{i=1}^{N_{p}}\left\{P_{t} g_{i s} \leq \tau\right\}\right) \geq 1-O_{\max }$.

Let $\gamma_{k}$ denote the instantaneous received SNR when the STx uses antenna $k$ for transmission. The instantaneous SEP, which we denote by $\operatorname{SEP}\left(\gamma_{k}\right)$, is given by [27, eq. (14)]

$$
\operatorname{SEP}\left(\gamma_{k}\right) \approx c_{1} \exp \left(-c_{2} \gamma_{k}\right)
$$

where $c_{1}$ and $c_{2}$ are modulation-specific constants. For MRC, $\gamma_{k}=P_{t}\left(\sum_{n=1}^{N_{r}} h_{n k}\right) / \sigma^{2}$, and for SC, $\gamma_{k}=$ $P_{t} \max \left\{h_{1 k}, \ldots, h_{N_{r} k}\right\} / \sigma^{2}$. The formula in (4) is exact for differential BPSK with $\left(c_{1}, c_{2}\right)=(0.5,1)$ and non-coherent binary frequency-shift-keying with $\left(c_{1}, c_{2}\right)=(0.5,0.5)$ [25]. It is a tight approximation for QPSK with $\left(c_{1}, c_{2}\right)=(0.5,0.6)$, for 8-PSK with $\left(c_{1}, c_{2}\right)=(0.6,0.18)$, and for 16-QAM with $\left(c_{1}, c_{2}\right)=(0.8,0.12)$.

When the STx transmits with power $P_{t}$, the SEP is strictly less than $c_{1}$. However, when it transmits with 0 power, i.e., $s=0$, we have $\gamma_{0}=0$. Therefore, from (4), the SEP equals $c_{1}<1$. Thus, choosing antenna 0 entails a worst-case penalty; it will ensure that the optimal TAS rule does not always select $s=0$ in order to trivially satisfy the interference constraint [8], [11]. We note that this approximation is consistent with the fact that for a constellation of size $M$, the SEP for $s=0$ is exactly $^{1} e_{0} \triangleq 1-(1 / M)<1$.

\footnotetext{
${ }^{1}$ For this case, the optimal decoder at the SRx declares any one of the $M$ symbols as its output with equal probability.
}

TAS Rule Definition: A TAS rule $\phi$ is a mapping from $\left(\mathbf{H}, \mathbf{G}_{\mathcal{A}}\right)$ to the set $\left\{0,1, \ldots, N_{t}\right\}$. The antenna selected is $s=\phi\left(\mathbf{H}, \mathbf{G}_{\mathcal{A}}\right)$. Since $\mathbf{H}$ and $\mathbf{G}_{\mathcal{A}}$ are random, so is $s$.

Finding the optimal TAS rule $\phi^{*}$ that minimizes the average SEP of the secondary system subject to the interference-outage constraint and given $\mathcal{A}$ can be mathematically stated as the following stochastic, constrained optimization problem $\mathcal{P}$ :

$$
\begin{aligned}
& \mathcal{P}: \min _{\phi} \mathbb{E}_{\mathbf{H}, \mathbf{G}_{\mathcal{A}}}\left[\operatorname{SEP}\left(\gamma_{s}\right)\right] \\
& \text { s.t. } \operatorname{Pr}(\overbrace{i=1}^{N_{p}}\left\{P_{t} g_{i s} \leq \tau\right\}) \geq 1-O_{\max }, \\
& s=\phi\left(\mathbf{H}, \mathbf{G}_{\mathcal{A}}\right) .
\end{aligned}
$$

\section{OPtimal TAS RULE AND ITS INTERFERENCE-OUTAGE PROBABILITY}

We now present the optimal TAS rule and derive its interference-outage probability.

\section{A. Optimal TAS Rule}

Let us first consider the case when the interference-outage constraint is inactive. Here, it is easy to see that the optimal TAS rule selects the antenna with the highest instantaneous SNR. It is given by

$$
s=\underset{k \in\left\{1,2, \ldots, N_{t}\right\}}{\arg \max }\left\{\gamma_{k}\right\} .
$$

We shall call this the unconstrained rule. Its interferenceoutage probability $O_{u}$ can be written as

$$
O_{u}=\operatorname{Pr}\left(\bigcup_{i=1}^{N_{p}}\left\{P_{t} g_{i s}>\tau\right\}\right)=\operatorname{Pr}\left(\bigcup_{i=1}^{N_{p}}\left\{P_{t} g_{i 1}>\tau\right\}\right),
$$

where the second equality follows because: (i) the antenna selected by the unconstrained rule does not depend on $\mathbf{G}_{\mathcal{A}}$, and (ii) $\cup_{i=1}^{N_{p}}\left\{P_{t} g_{i k}>\tau\right\}$, for $1 \leq k \leq N_{t}$, are statistically identical. Applying the De Morgan's law and using the fact that $g_{11}, \ldots, g_{N_{p} 1}$ are independent, we get

$$
O_{u}=1-\operatorname{Pr}\left(\stackrel{N}{i=1}_{N_{p}}^{\cap}\left\{P_{t} g_{i 1} \leq \tau\right\}\right)=1-\prod_{i=1}^{N_{p}} F_{g_{i}}\left(\frac{\tau}{P_{t}}\right),
$$

where $F_{g_{i}}(\cdot)$ denotes the CDF of $g_{i k}$, for $1 \leq k \leq N_{t}$. Clearly, the unconstrained rule is optimal when $O_{u} \leq O_{\max }$. We shall call this region as the unconstrained region.

However, when $O_{u}>O_{\max }$, which we shall refer to as the constrained region, the above rule does not satisfy the interference-outage constraint and cannot be optimal for the problem $\mathcal{P}$. To develop the optimal solution in this region, we introduce the following notations. When the STx transmits with the $k^{\text {th }}$ antenna, let $L_{k}(\mathcal{A})$ denote the event that $P_{t} g_{i k} \leq$ $\tau$ at all the PRxs whose CSI is available at the STx, and let $D_{k}\left(\mathcal{A}^{c}\right)$ denote the event that $P_{t} g_{i k} \leq \tau$ at all the PRxs whose CSI is not available at the STx. Thus,

$L_{k}(\mathcal{A}) \triangleq \bigcap_{i \in \mathcal{A}}\left\{P_{t} g_{i k} \leq \tau\right\}$ and $D_{k}\left(\mathcal{A}^{c}\right) \triangleq \bigcap_{i \in \mathcal{A}^{c}}\left\{P_{t} g_{i k} \leq \tau\right\}$.

Let $\alpha_{0}\left(\mathcal{A}^{c}\right) \triangleq \operatorname{Pr}\left(D_{0}\left(\mathcal{A}^{c}\right)\right)=1$ and, for $1 \leq k \leq N_{t}$,

$$
\alpha_{k}\left(\mathcal{A}^{c}\right) \triangleq \operatorname{Pr}\left(D_{k}\left(\mathcal{A}^{c}\right)\right)=\prod_{i \in \mathcal{A}^{c}} F_{g_{i}}\left(\frac{\tau}{P_{t}}\right) .
$$


In terms of these notations, the optimal TAS rule is as follows.

Result 1: Given $\mathcal{A}$, the optimal rule $\phi^{*}$ selects antenna $s^{*}=\phi^{*}\left(\mathbf{H}, \mathbf{G}_{\mathcal{A}}\right)$ that is given by

$$
s^{*}= \begin{cases}\underset{k \in\left\{1,2, \ldots, N_{t}\right\}}{\arg \max }\left\{\gamma_{k}\right\}, & O_{u} \leq O_{\max }, \\ \underset{k \in\left\{0,1, \ldots, N_{t}\right\}}{\arg \min }\left\{y_{k}-\lambda \alpha_{k}\left(\mathcal{A}^{c}\right) I_{\left\{L_{k}(\mathcal{A})\right\}}\right\}, & O_{u}>O_{\max },\end{cases}
$$

where $y_{k}=\operatorname{SEP}\left(\gamma_{k}\right)$. Here, $\lambda>0$ is chosen such that the interference-outage constraint in (6) is met with equality. Furthermore, such a choice of $\lambda$ always exists.

Proof: The proof is given in Appendix A.

Comments: In Section III-D, we discuss how to compute $\lambda$ with low complexity. We see that both $\mathcal{A}$ and $\mathcal{A}^{c}$ affect the optimal rule. The STx can satisfy the interference-outage constraint even with partial CSI because of the stochastic nature of the constraint. This is unlike the peak-interference constraint, which can be satisfied by the STx only with full CSI. Another feature of the optimal rule is that the STx only needs to know if the STx-PRx channel power gains exceeds $\tau / P_{t}$ or not. It can be shown that the above rule applies even when the channel gains are correlated; only the value of $\lambda$ changes.

\section{B. Interpretation of the Optimal Rule in Constrained Region}

We first introduce the following terminology. We say that an STx antenna $k \in\left\{1,2, \ldots, N_{t}\right\}$ is $\mathcal{A}$-outage-compatible if $P_{t} g_{i k} \leq \tau$, for $i \in \mathcal{A}$, i.e., $I_{\left\{L_{k}(\mathcal{A})\right\}}=1$. Otherwise, we say that it is outage-incompatible, which means that it causes an interference power greater than $\tau$ at at least one of the PRxs. To keep the notation simple, we shall use $\alpha_{k}$ and $L_{k}$ instead of $\alpha_{k}\left(\mathcal{A}^{c}\right)$ and $L_{k}(\mathcal{A})$ henceforth.

Another interpretation of (12) is that the optimal rule selects the antenna with the smallest metric, where the metric of the $k^{\text {th }}$ STx antenna is $y_{k}-\lambda \alpha_{k} I_{\left\{L_{k}\right\}}$. From (11), we see that $\alpha_{1}=\cdots=\alpha_{N_{t}} \triangleq \alpha$. Thus, for an antenna $k \in$ $\left\{1,2, \ldots, N_{t}\right\}$, the metric is $y_{k}$ if it is outage-incompatible and $y_{k}-\lambda \alpha$ if it is $\mathcal{A}$-outage-compatible. For full CSI $\alpha=$ $\operatorname{Pr}\left(D_{1}(\varnothing)\right)=1$. For partial CSI, the factor $\alpha<1$ can be interpreted as a CSI penalty because it increases the metric of an $\mathcal{A}$-outage-compatible antenna. Thus, as $\alpha$ decreases, the penalty increases. The metric of antenna 0 is equal to $c_{1}-\lambda$. Thus, the optimal rule selects $s^{*} \neq 0$ if and only if there is at least one antenna $1,2, \ldots, N_{t}$ whose metric is smaller than $c_{1}-\lambda$. Else, $s^{*}=0$.

Fig. 2 illustrates the metric for $N_{p}=2$ and brings out the key role that partial CSI plays in it. It plots the metric of antenna $k$ for full CSI and partial CSI. With full CSI, i.e., $\mathcal{A}=$ $\{1,2\}$, we have $\alpha=1$ and the metric depends on both $g_{1 k}$ and $g_{2 k}$. It is equal to $y_{k}-\lambda$ if $P_{t} g_{1 k} \leq \tau$ and $P_{t} g_{2 k} \leq \tau$; else, it is equal to $y_{k}$. With partial CSI and $\mathcal{A}=\{1\}$, the metric depends on $g_{1 k}$ and the statistics of $g_{2 k}$ through $\alpha=\operatorname{Pr}\left(P_{t} g_{2 k} \leq \tau\right)<$ 1. It is equal to $y_{k}-\lambda \alpha$ if $P_{t} g_{1 k} \leq \tau$; else, it is equal to $y_{k}$. A similar behavior is seen for $\mathcal{A}=\{2\}$.

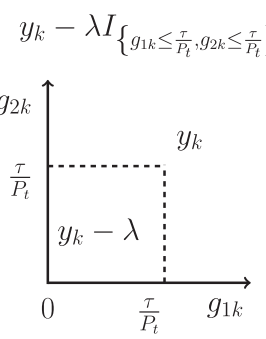

a) $\mathcal{A}=\{1,2\}$

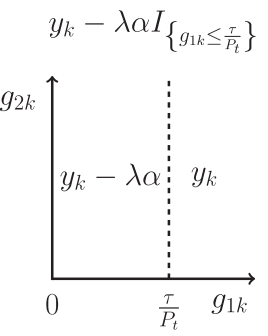

b) $\mathcal{A}=\{1\}$

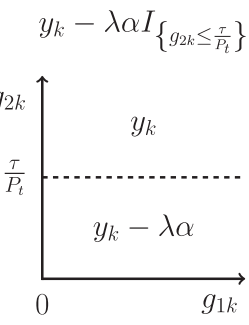

c) $\mathcal{A}=\{2\}$
Fig. 2. Metric of the antenna $k$ for full and partial CSI scenarios $\left(N_{p}=2\right)$.

\section{Behavior of the Optimal TAS Rule when $\lambda \geq c_{1}$}

We now consider the case when $\lambda \geq c_{1}$. This scenario arises when $\tau$ or $O_{\max }$ are small. In this case, the metric $c_{1}-\lambda$ of antenna 0 is always non-positive while the metric of an outageincompatible antenna $k$ is strictly positive. It can be shown that the optimal rule simplifies to

$$
s^{*}= \begin{cases}\underset{k \in \mathcal{T}\left(\mathbf{H}, \mathbf{G}_{\mathcal{A}}\right)}{\arg \max }\left\{\gamma_{k}\right\}, & \text { if } \mathcal{T}\left(\mathbf{H}, \mathbf{G}_{\mathcal{A}}\right) \neq \varnothing, \\ 0, & \text { else, }\end{cases}
$$

where $\mathcal{T}\left(\mathbf{H}, \mathbf{G}_{\mathcal{A}}\right)=\left\{k \mid \cap_{i \in \mathcal{A}}\left\{P_{t} g_{i k} \leq \tau\right\}, \gamma_{k}>\eta\right\}$ is the set of $\mathcal{A}$-outage compatible antennas whose SNR exceeds $\eta=-\ln \left(1-\left[\lambda(1-\alpha) / c_{1}\right]\right) / c_{2}$. Notice that the impact of the interference-outage constraint and the partial CSI is entirely captured by a single parameter $\eta$.

\section{Interference-Outage Probability of the Optimal TAS Rule}

We now derive an exact expression and a bound for the interference-outage probability of the optimal rule $\phi^{*}$. Since $y_{1}, \ldots, y_{N_{t}}$ are identically distributed, we denote their marginal complementary CDF (CCDF) and probability density function $(\mathrm{PDF})$ by $F_{y}^{c}(\cdot)$ and $f_{y}(\cdot)$, respectively. Let

$$
p_{l}=\operatorname{Pr}\left(L_{k}\right)=\prod_{i \in \mathcal{A}} F_{g_{i}}\left(\frac{\tau}{P_{t}}\right), \text { for } 1 \leq k \leq N_{t} .
$$

Lemma 1: The interference-outage probability $O_{\lambda}$ of $\phi^{*}$ is

$$
\begin{aligned}
O_{\lambda}=1 & -\Psi_{0}-N_{t}\left(1-O_{u}\right) \int_{0}^{c_{1}-\lambda(1-\alpha)} f_{y}(x) \\
& \times\left[\left(1-p_{l}\right) F_{y}^{c}(x-\lambda \alpha)+p_{l} F_{y}^{c}(x)\right]^{N_{t}-1} d x,
\end{aligned}
$$

where $\Psi_{0}=\left[p_{l} F_{y}^{c}\left(c_{1}-\lambda(1-\alpha)\right)+\left(1-p_{l}\right) F_{y}^{c}\left(c_{1}-\lambda\right)\right]^{N_{t}}$. Furthermore, $O_{\lambda}$ is upper bounded by

$$
\begin{array}{r}
O_{\lambda} \leq(1-a)\left(1-\Psi_{0}\right)+a\left(\left[1-p_{l}+p_{l} F_{y}^{c}(\lambda \alpha)\right]^{N_{t}}\right. \\
\left.-\left[\left(1-p_{l}\right) F_{y}^{c}\left(c_{1}-\lambda\right)+p_{l} F_{y}^{c}(\lambda \alpha)\right]^{N_{t}}\right),
\end{array}
$$

where $a=\left(1-O_{u}\right) / p_{l}$.

Proof: The proof is given in Appendix B.

To obtain $\lambda$, one needs to numerically solve the equation $O_{\lambda}=O_{\max }$. We present an alternate, lower complexity approach below. In it, the upper bound in (16) is instead equated with $O_{\max }$. This can be shown to yield an upper bound 
for $\lambda$. In Section V, we shall see that using this upper bound has a negligible impact on performance.

For $\lambda \geq c_{1}$ and $0<x \leq c_{1}-\lambda(1-\alpha)$, we know $x-\lambda \alpha<0$. Hence, $F_{y}^{c}(x-\lambda \alpha)=1$. Substituting this in (15) yields the following closed-form expression for $O_{\lambda}$ for $\lambda \geq c_{1}$ :

$$
O_{\lambda}=(1-a)\left(1-\left[1-p_{l}+p_{l} F_{y}^{c}\left(c_{1}-\lambda(1-\alpha)\right)\right]^{N_{t}}\right) \text {. }
$$

For example, for Rayleigh fading, $h_{n k}$ and $g_{i k}$ are exponential RVs. Let $\Omega \triangleq P_{t} \mu_{h} / \sigma^{2}$. The CDF of $g_{i k}$ is $F_{g_{i}}(x)=1-$ $\exp \left(-x / \mu_{i}\right)$, for $x \geq 0$. When the SRx employs MRC, the $\mathrm{CCDF}$ of $y_{1}$ is given by

$$
F_{y}^{c}(x)=1-\left(\frac{x}{c_{1}}\right)^{\frac{1}{c_{2} \Omega}} \sum_{n=0}^{N_{r}-1} \frac{1}{n !}\left(-\frac{1}{c_{2} \Omega} \ln \left(\frac{x}{c_{1}}\right)\right)^{n},
$$

for $x \in\left(0, c_{1}\right]$. Instead, if the SRx employs SC, then the CCDF of $y_{1}$ is given by $F_{y}^{c}(x)=\left[1-\left(x / c_{1}\right)^{\frac{1}{c_{2} \Omega}}\right]^{N_{r}}$, for $x \in\left(0, c_{1}\right]$. Substituting these CCDFs in (15) yields the corresponding expressions for $O_{\lambda}$.

\section{E. Extreme Cases}

To gain insights, we consider the following extreme cases:

1) Full CSI: Here, $L_{k}=\cap_{i=1}^{N_{p}}\left\{P_{t} g_{i k} \leq \tau\right\}, \alpha=1$, and $p_{l}=1-O_{u}$. Substituting these in (12), we get

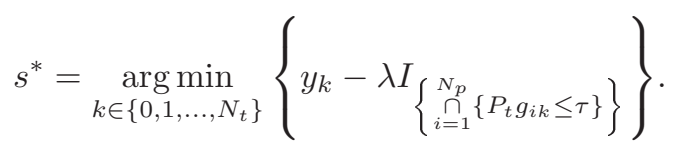

For $\lambda=c_{1}$, the metric of antenna 0 is equal to zero, the metric of antenna $k \in\left\{1, \ldots, N_{t}\right\}$ is $y_{k}-c_{1} \leq 0$ if it is $\mathcal{A}$-outage-compatible and is $y_{k}>0$ otherwise. Therefore, as $\lambda \rightarrow c_{1}$, the optimal rule selects the antenna with the highest instantaneous SNR from the set of $\mathcal{A}$-outagecompatible antennas so long as it is not empty. Else, $s^{*}=$ $\arg \min \left\{y_{0}-\lambda, y_{1}, \ldots, y_{N_{t}}\right\}$. In this region, its interferenceoutage probability can be written in the following closed form:

$$
O_{\lambda}=O_{u}^{N_{t}}-O_{u}^{N_{t}}\left(F_{y}^{c}\left(c_{1}-\lambda\right)\right)^{N_{t}} .
$$

From (20), we see that $O_{\lambda}$ increases as $c_{1}$ or $O_{u}$ increases. For Rayleigh fading and SC, equating (20) with $O_{\max }$ results in the following closed-form expression for $\lambda$ that holds for $O_{u}^{N_{t}}>O_{\max }$ :

$$
\lambda=c_{1}-c_{1}\left(1-\left[1-\frac{O_{\max }}{O_{u}^{N_{t}}}\right]^{\frac{1}{N_{t} N_{r}}}\right)^{c_{2} \Omega} .
$$

Furthermore, using (16), for $N_{t}=2$, we can show that

$$
\lambda \leq c_{1}\left(1-\left[\frac{O_{\max }-O_{u}^{2}}{2 O_{u}\left(1-O_{u}\right)}\right]^{\frac{1}{N_{r}}}\right)^{c_{2} \Omega} .
$$

We see that $\lambda$ increases as $c_{1}$ increases or it decreases as $O_{\max }$ increases.

Note: The expression for $O_{\lambda}$ in [10, eq. (18)], which applies to a single PRx, is a special case of (20). Furthermore, the expressions for $\lambda$ in [10, eq. (19)] and [10, eq. (15)], which apply to a single PRx and $N_{r}=1$, are special cases of (21) and (22), respectively.
TABLE I

\section{KEY NOTATIONS}

\begin{tabular}{ll}
\hline$N_{t}$ & Number of transmit antennas at the STx \\
\hline$N_{r}$ & Number of receive antennas at the SRx \\
\hline $\mathbf{H}=\left[h_{n k}\right]$ & STx-SRx channel power gain matrix \\
\hline$N_{p}$ & Number of PRxs \\
\hline$N_{a}$ & Number of PRxs whose CSI is available \\
\hline $\mathcal{A}$ & Indices of PRxs whose CSI is available \\
\hline $\mathbf{G}_{\mathcal{A}}=\left[g_{i k}\right]$, for $i \in \mathcal{A}$ & STx-PRx channel power gain sub-matrix \\
\hline$s$ & Index of the STx antenna selected \\
\hline$c_{1}, c_{2}$ & Modulation parameters \\
\hline$\gamma_{k}$ & Instantaneous SNR of antenna $k$ \\
\hline$y_{k}$ & Instantaneous SEP of antenna $k$ \\
\hline$P_{t}, \Omega$ & Fixed transmit power, SNR \\
\hline$\tau$ & Interference power threshold \\
\hline$O_{\max }$ & Interference-outage probability threshold \\
\hline$\lambda$ & Penalization parameter \\
\hline$O_{u}$ & Unconstrained interference-outage probability \\
\hline$L_{k}$ & Event that $P_{t} g_{i k} \leq \tau$ for all $i \in \mathcal{A}$ \\
\hline$D_{k}$ & Event that $P_{t} g_{i k} \leq \tau$ for all $i \in \mathcal{A}^{c}$ \\
\hline$p_{l}, \alpha$ & Probability of $L_{k}$, probability of $D_{k}$ \\
\hline
\end{tabular}

2) $\operatorname{No} \operatorname{CSI}(\mathcal{A}=\varnothing)$ : In this case, the optimal rule in the constrained region becomes

$$
s^{*}=\arg \min \left\{y_{0}-\lambda, y_{1}-\lambda \alpha, \ldots, y_{N_{t}}-\lambda \alpha\right\} .
$$

Substituting $y_{0}=c_{1}$ and $y_{k}=c_{1} \exp \left(-c_{2} \gamma_{k}\right)$, for $1 \leq k \leq$ $N_{t}$, in (23), we get

$$
s^{*}= \begin{cases}0, & \text { if } \gamma_{1} \leq \eta, \ldots, \gamma_{N_{t}} \leq \eta, \\ \underset{k \in\left\{1,2, \ldots, N_{t}\right\}}{\arg \max }\left\{\gamma_{k}\right\}, & \text { else, }\end{cases}
$$

where $\eta=-\ln \left(1-\left[\lambda(1-\alpha) / c_{1}\right]\right) / c_{2}$ (cf. Section IIIC). Hence, $\phi^{*}$ selects $s^{*}=0$ if $\gamma_{1}, \ldots, \gamma_{N_{t}}$ are all less than or equal to $\eta$. Else, the antenna with the highest instantaneous SNR is selected. For Rayleigh fading and SC, the interference-outage probability of this rule is equal to $O_{u}-O_{u}(1-\exp (-\eta / \Omega))^{N_{t} N_{r}}$. Equating this with $O_{\max }$ yields the following expression for $\eta$ directly in terms of $O_{\max }$, $O_{u}$, and $\Omega$ :

$$
\eta=-\Omega \ln \left(1-\left[1-\frac{O_{\max }}{O_{u}}\right]^{\frac{1}{N_{t} N_{r}}}\right) .
$$

\section{SEP ANALYSis OF THE OPtimal TAS RULE}

We now derive a general expression for the average SEP, which we denote by $\overline{\mathrm{SEP}}$. We also investigate a special case and two extreme cases to gain more insights.

Result 2: For the optimal rule, $\overline{\mathrm{SEP}}=T_{1}+T_{2}+T_{3}$, where

$$
\begin{aligned}
T_{1}= & e_{0} \Psi_{0}, \\
T_{2}= & N_{t} p_{l} \int_{0}^{c_{1}-\lambda(1-\alpha)} x f_{y}(x) \\
& \times\left[\left(1-p_{l}\right) F_{y}^{c}(x-\lambda \alpha)+p_{l} F_{y}^{c}(x)\right]^{N_{t}-1} d x, \\
T_{3}= & N_{t}\left(1-p_{l}\right) \int_{0}^{c_{1}-\lambda} x f_{y}(x) \\
& \times\left[\left(1-p_{l}\right) F_{y}^{c}(x)+p_{l} F_{y}^{c}(x+\lambda \alpha)\right]^{N_{t}-1} d x,
\end{aligned}
$$

where $\Psi_{0}$ is given in Lemma 1 . 
Proof: The proof is given in Appendix C.

The above expression applies to many modulation schemes, any number of PRxs, to any number of antennas, and to the general class of continuous fading models. In the unconstrained region, i.e., $\lambda=0$, it simplifies to $\overline{\mathrm{SEP}}=$ $\int_{0}^{c_{1}}\left[F_{y}^{c}(x)\right]^{N_{t}} d x$.

Insights: The first term $T_{1}$ is the average SEP due to $s^{*}=0$. It increases as $M$ or $\lambda$ increases. As the CSI of more STx-PRx links becomes available, i.e., $\mathcal{A}$ becomes larger, $\alpha$ increases and finally becomes one for full CSI. Consequently, since the CCDF $F_{y}^{c}(\cdot)$ is a monotonically non-increasing function, the $F_{y}^{c}\left(c_{1}-\lambda(1-\alpha)\right)$ term in $T_{1}$ decreases as $\alpha$ increases and becomes zero for full CSI. $T_{2}$ is the average SEP when an $\mathcal{A}$-outage-compatible antenna is selected. It increases as $\lambda$ increases for $\lambda<c_{1}$ and decreases as $\lambda$ increases for $\lambda \geq c_{1}$. The third term $T_{3}$ is the average SEP when an outageincompatible antenna is selected. Unlike $T_{1}, T_{3}$ decreases as $\lambda$ increases and becomes zero when $\lambda=c_{1}$.

\section{A. Special Case: Rayleigh Fading and SC}

a) For $\lambda=0, \overline{\text { SEP }}$ simplifies to

$$
\overline{\mathrm{SEP}}=c_{1} c_{2} \Omega \sum_{k=0}^{N_{t} N_{r}}\left(\begin{array}{c}
N_{t} N_{r} \\
k
\end{array}\right) \frac{(-1)^{k}}{k+c_{2} \Omega} .
$$

b) For $0<\lambda<c_{1}$, the sum $T_{2}+T_{3}$ takes the following simplified form:

$$
\begin{aligned}
T_{2}+ & T_{3} \\
= & \frac{N_{t} N_{r} c_{1}}{c_{2} \Omega} \sum_{k=0}^{N_{t}-1} \sum_{i=0}^{N_{r} k} \sum_{j=0}^{N_{r}\left(N_{t}-k\right)-1}\left(\begin{array}{c}
N_{t}-1 \\
k
\end{array}\right)\left(\begin{array}{c}
N_{r} k \\
i
\end{array}\right) \\
& \times\left(\begin{array}{c}
N_{r}\left(N_{t}-k\right)-1 \\
j
\end{array}\right)\left[\left(1-p_{l}\right)^{k} p_{l}^{N_{t}-k} \psi_{j+1, i}\right. \\
& \left.+\left(1-p_{l}\right)^{N_{t}-k} p_{l}^{k} \psi_{i, j+1}\right](-1)^{(i+j)} \\
& +N_{t} N_{r} c_{1} \sum_{k=0}^{N_{t}-1} \sum_{i=0}^{N_{r}(k+1)-1}\left(\begin{array}{c}
N_{r}(k+1)-1 \\
i
\end{array}\right) \\
& \times \frac{\left(\begin{array}{c}
N_{t}-1 \\
k
\end{array}\right)\left(\frac{\lambda \alpha}{c_{1}}\right)^{1+\frac{i+1}{c_{2} \Omega}}\left(1-p_{l}\right)^{N_{t}-k-1} p_{l}^{k+1}}{(-1)^{i}\left(c_{2} \Omega+i+1\right)},
\end{aligned}
$$

where

$$
\begin{aligned}
\psi_{k_{1}, k_{2}} & =\int_{0}^{1-\frac{\lambda}{c_{1}}}\left(x+\frac{\lambda \alpha}{c_{1}}\right)^{\frac{k_{1}}{c_{2} \Omega}} x^{\frac{k_{2}}{c_{2} \Omega}} d x \\
& \approx\left(1-\frac{\lambda}{c_{1}}\right) \sum_{l=1}^{n_{g}} w_{l}\left(v_{l}+\frac{\lambda \alpha}{c_{1}}\right)^{\frac{k_{1}}{c_{2} \Omega}} v_{l}^{\frac{k_{2}}{c_{2} \Omega}}
\end{aligned}
$$

$v_{l}=\left(1-\left(\lambda / c_{1}\right)\right) x_{l}$, and $x_{l}$ and $w_{l}$ are the $n_{g}$ abscissas and weights, respectively, for Gaussian integration of moments [28, pp. 921 and 922]. Furthermore, for $\lambda \in\left(c_{1} / 2, c_{1}\right), \psi_{k_{1}, k_{2}}$ can be written exactly in terms of the following infinite series [29]:

$$
\psi_{k_{1}, k_{2}}=\sum_{m=0}^{\infty} \frac{\Gamma\left(\frac{k_{1}}{c_{2} \Omega}+1\right)\left(\frac{\lambda \alpha}{c_{1}}\right)^{\frac{k_{1}}{c_{2} \Omega}-m}\left(1-\frac{\lambda}{c_{1}}\right)^{\frac{k_{2}}{c_{2} \Omega}+m+1}}{\Gamma\left(\frac{k_{1}}{c_{2} \Omega}-m+1\right) m !\left(\frac{k_{2}}{c_{2} \Omega}+m+1\right)} .
$$

It turns out that five terms are sufficient to compute the above summation accurately. We note that the expression in [10, eq. (27)] is a special case of (33) for full CSI $(\alpha=1)$.

b) For $\lambda \geq c_{1}, T_{3}=0$ and $T_{2}$ simplifies to:

$$
\begin{aligned}
T_{2}= & N_{t} N_{r} c_{1} \sum_{k=0}^{N_{t}-1} \sum_{i=0}^{N_{r}(k+1)-1}\left(\begin{array}{c}
N_{t}-1 \\
k
\end{array}\right)\left(\begin{array}{c}
N_{r}(k+1)-1 \\
i
\end{array}\right) \\
& \times \frac{\left(1-p_{l}\right)^{N_{t}-k-1} p_{l}^{k+1}\left(1-\frac{\lambda(1-\alpha)}{c_{1}}\right)^{1+\frac{(i+1)}{c_{2} \Omega}}}{(-1)^{i}\left(c_{2} \Omega+i+1\right)}
\end{aligned}
$$

This term, as we saw before, decreases as $\lambda$ increases.

\section{B. Extreme Cases}

For full CSI, $\overline{\mathrm{SEP}}$ is obtained by substituting $\alpha=1$ and $p_{l}=$ $1-O_{u}$ in the above expressions. No further simplifications occur. However, for no CSI, $\overline{\mathrm{SEP}}$ does simplify as follows for Rayleigh fading and SC:

$$
\begin{aligned}
\overline{\mathrm{SEP}}=e_{0} & \left(1-\frac{O_{\max }}{O_{u}}\right)+N_{t} N_{r} c_{1} e^{-c_{2} \eta} \\
& \times \sum_{k=0}^{N_{t} N_{r}-1}\left(\begin{array}{c}
N_{t} N_{r}-1 \\
k
\end{array}\right) \frac{(-1)^{k} e^{-\frac{(k+1) \eta}{\Omega}}}{c_{2} \Omega+k+1} .
\end{aligned}
$$

We can clearly see the dependence of SEP on system parameters such as $N_{t}, N_{r}, M, O_{\max }$, and $\Omega$. The first term, which is the average SEP due to $s^{*}=0$, is independent of $N_{t}$ and $N_{r}$, and increases as $O_{\max }$ decreases. On the other hand, the second term, which is the average SEP when one among the antennas $1,2, \ldots, N_{t}$ is selected, increases as $c_{1}$ increases or as $c_{2}$ decreases. It decreases exponentially as $\eta$ increases.

\section{Numerical Results and Performance BENCHMARKING}

We now present Monte Carlo simulations, which simulate the transmit and receive chains and use $10^{6}$ data symbols, to verify the analytical results and study the impact of the partial CSI. We also benchmark the performance of the optimal TAS rule with several other TAS rules. We set $\mu_{h}=1$ and $\sigma^{2}=1$. To capture the non-identical nature of the channel power gains from the STx to different PRxs, we set $\mu_{i}=$ $\kappa^{i}$, for $i \in\left\{1,2, \ldots, N_{p}\right\}$, where $\kappa \leq 1$. The farther $\kappa$ is from 1, the more non-identical are the gains. We show results for Rayleigh fading.

For full CSI, Fig. 3 plots the average SEP of the optimal rule as a function of the interference power threshold $\tau$ for different values of $O_{\max }$ and $N_{t}$. The analytical and simulation results match well. The behavior depends on the region of operation of the system as follows: (i) Constrained region $\left(O_{u}>O_{\max }\right)$ : $\overline{\mathrm{SEP}}$ decreases as $\tau$ increases because more interference power is allowed at each PRx. It also decreases as $O_{\max }$ increases because the constraint is relaxed. (ii) Unconstrained region $\left(O_{u} \leq O_{\max }\right)$ : Here, $\overline{\mathrm{SEP}}$ becomes the same as that of the unconstrained rule and is independent of $\tau$ and $O_{\max }$. This happens for $\tau \geq 14.1 \mathrm{~dB}$ at $O_{\max }=0.1$ and for $\tau \geq 15.0 \mathrm{~dB}$ at $O_{\max }=0.05$ for all $N_{t}$ and $N_{r}$. The value of $\tau$ where the unconstrained region starts can be solved by equating 


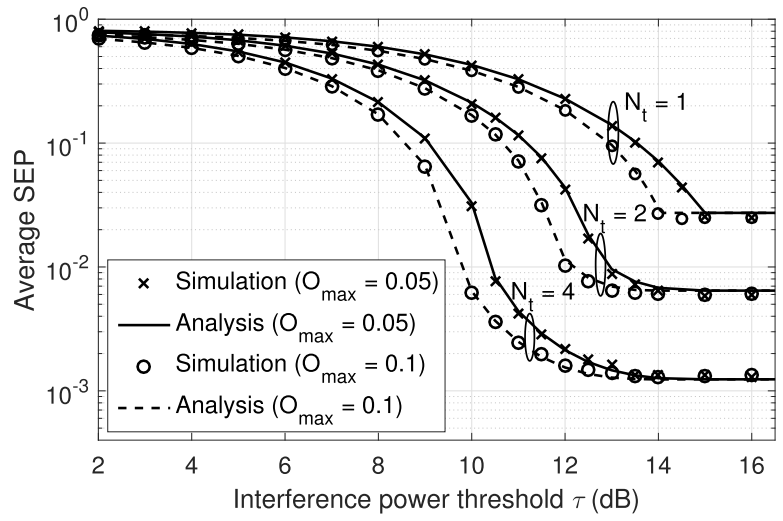

Fig. 3. Full CSI: Average SEP as a function of the interference power threshold $\tau$ for different values of $O_{\max }$ and $N_{t}\left(N_{p}=2, N_{r}=3 P_{t}=\right.$ $10 \mathrm{~dB}, \kappa=0.9, \mathrm{MRC}$, and 8-PSK with $c_{1}=0.6$ and $c_{2}=0.18$ ).

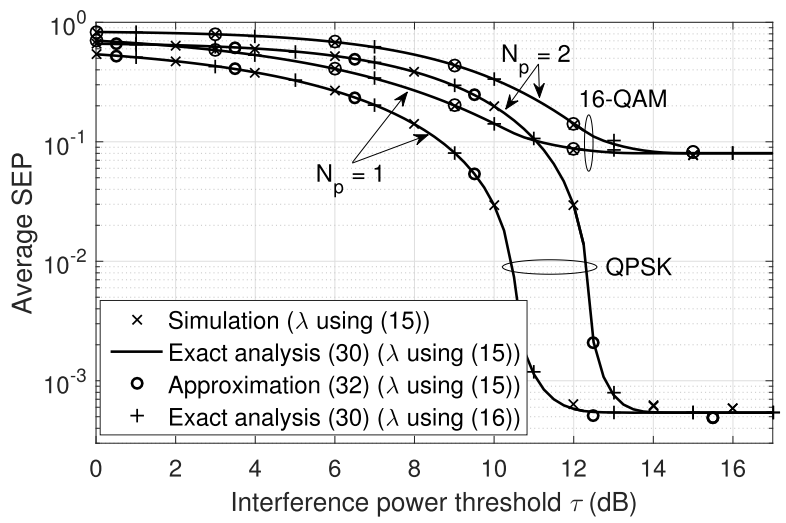

Fig. 4. Effect of $N_{p}$ for full CSI: Average SEP as a function of $\tau$ for different number of PRxs $\left(O_{\max }=0.1, N_{t}=2, N_{r}=3, P_{t}=10 \mathrm{~dB}\right.$, $\kappa=1$, SC, QPSK with $c_{1}=0.5$ and $c_{2}=0.6$, and 16-QAM with $c_{1}=0.8$ and $c_{2}=0.12$ ).

$O_{u}$ in (10) to $O_{\max }$. We observe that $\overline{\mathrm{SEP}}$ decreases as $N_{t}$ increases, which shows that TAS exploits spatial diversity even though it uses only one RF chain.

Fig. 4 investigates the impact of the number of PRxs, $N_{p}$, for full CSI. It also compares two modulation schemes. It plots the average SEP as a function of $\tau$ for the special case of identical STx-PRx channels when the SRx uses SC. The exact analytical expression in (30) and its approximation in (32) (with $n_{g}=5$ ) are shown. They both match the simulation results well. In the constrained region, $\overline{\mathrm{SEP}}$ increases as $N_{p}$ increases. This is intuitive as the interference power needs to be controlled at more PRxs. In this case, the value of $\tau$ at which the unconstrained region starts can be shown to be equal to $-P_{t} \mu_{g} \ln \left(1-\left(1-O_{\max }\right)^{\left(1 / N_{p}\right)}\right)$, which depends on $N_{p}$ but not the modulation scheme. It is equal to $13.6 \mathrm{~dB}$ and $14.7 \mathrm{~dB}$ for $N_{p}=1$ and 2, respectively. In the unconstrained region, $\overline{\text { SEP }}$ saturates to the value given by (29), which depends on the modulation scheme but not $N_{p}$. We see that in both regions, it increases as $M$ increases. Fig. 4 also compares $\overline{\text { SEP }}$ when $\lambda$ is obtained by equating the exact $O_{\lambda}$ in (15) and when it is obtained by equating its upper bound in (16) to $O_{\max }$. We see that the two curves are indistinguishable.

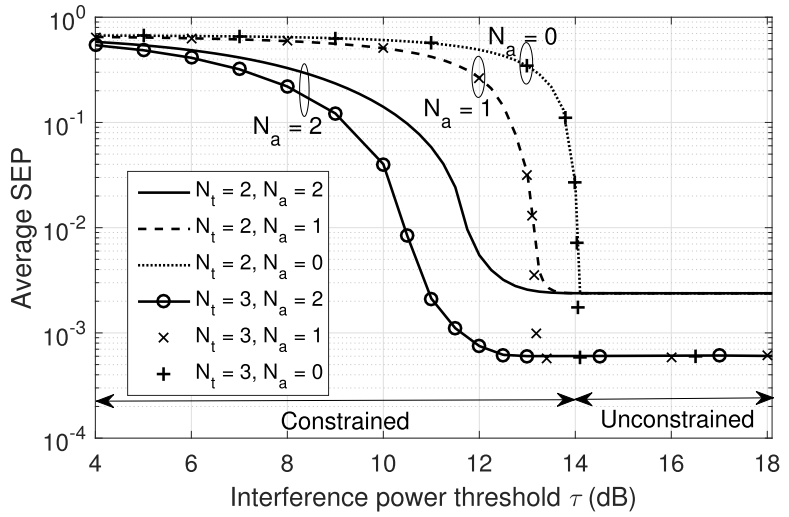

Fig. 5. Effect of partial CSI: Average SEP as a function of $\tau$ for different values of $N_{a}$ and $N_{t}\left(N_{p}=2, N_{r}=2, P_{t}=10 \mathrm{~dB}, \kappa=0.9\right.$, SC, and QPSK with $c_{1}=0.5$ and $c_{2}=0.6$ ).

\section{A. Impact of Partial CSI}

For partial CSI, we show results that are averaged over all possible subsets of PRxs for which the STx has CSI, which are taken to be equally likely. Fig. 5 plots the average SEP as a function of $\tau$ with full, partial, and no CSI at the STx for $N_{p}=2$ and two values of $N_{t}$. All the curves enter the unconstrained region at $\tau=14.1 \mathrm{~dB}$, regardless of the values of $N_{t}$ and $N_{a}$. (i) Constrained region $(\tau<14.1 \mathrm{~dB})$ : Here, for a given $N_{t}, \overline{\text { SEP }}$ increases as the STx-PRx CSI decreases. With full CSI, we see that $\overline{\mathrm{SEP}}$ decreases as $N_{t}$ increases for all values of $\tau$. With partial or no CSI, the trends are different. $\overline{\mathrm{SEP}}$ is insensitive to $\tau$ for small $\tau$. It appreciably decreases as $N_{t}$ increases from 2 to 3 only when $\tau \geq 13.1 \mathrm{~dB}$ for partial CSI and $\tau \geq 14.05 \mathrm{~dB}$ for no CSI. (ii) Unconstrained region $(\tau \geq 14.1 \mathrm{~dB})$ : Here, for a given $N_{t}$, $\overline{\mathrm{SEP}}$ saturates to a value that is independent of $N_{a}$, which is the average SEP of the unconstrained rule. It decreases exponentially as $N_{t}$ increases.

To understand the insensitivity of SEP to $\tau$, Fig. 6 plots the probability of $s=0$ as a function of $\tau$ for full, partial, and no CSI scenarios. The probability of $s=0$ for partial CSI and no CSI is larger than that for full CSI since the STx chooses $s=0$ more often to meet the interference-outage constraint when it has less CSI. With full CSI, we see that the probability of $s=0$ decreases exponentially as $\tau$ increases. However, this is not so for partial CSI and no CSI. It is insensitive to $\tau$ for $\tau \leq 10 \mathrm{~dB}$, which contributes to the insensitivity of $\overline{\mathrm{SEP}}$ to $\tau$; it decreases exponentially for larger values of $\tau$.

\section{B. Performance Benchmarking}

We now compare the performance of the optimal rule with several other TAS rules considered in the literature. In order to ensure as comprehensive a comparison as possible, we also show results for rules that were originally proposed for single PRx. We do so by designing them on the basis of the maximum channel power gain among the links from the STx to the $N_{a}$ PRxs whose CSI is available. For the $k^{\text {th }}$ STx antenna, let $g_{k} \triangleq \max _{i \in \mathcal{A}}\left\{g_{i k}\right\}$.

1) Enhanced Minimum Interference (EMI) Rule [12]: Among the antennas $1, \ldots, N_{t}$, it selects the one with 


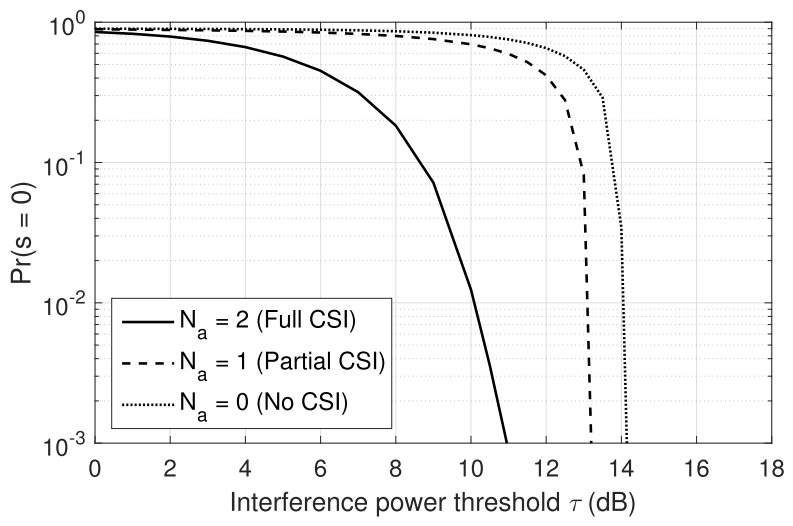

Fig. 6. Effect of partial CSI for $N_{p}=2$ : Probability of zero transmit power option $(s=0)$ being selected as a function of $\tau$ for different values of $N_{a}$ $\left(O_{\max }=0.1, N_{t}=4, N_{r}=1, P_{t}=10 \mathrm{~dB}, \kappa=1\right.$, and 16QAM with $c_{1}=0.8$ and $c_{2}=0.12$ ).

the smallest $g_{k}$. However, it selects antenna 0 when $g_{1}, \ldots, g_{N_{t}}$ exceed a threshold $\beta$. It is given by

$$
s= \begin{cases}0, & \text { if } g_{1} \geq \beta, \ldots, g_{N_{t}} \geq \beta, \\ \underset{k \in\left\{1, \ldots, N_{t}\right\}}{\arg \min }\left\{g_{k}\right\}, & \text { else. }\end{cases}
$$

2) Enhanced Maximum Signal Power to Leak Interference Power Ratio (EMSLIR) Rule [12]: Among the antennas $1, \ldots, N_{t}$, it selects the one with the largest ratio of the instantaneous SNR $\gamma_{k}$ to the worst case STx-PRx channel power gain $g_{k}$. However, it selects antenna 0 when all ratios are below a threshold $\xi$. It is given by

$$
s= \begin{cases}0, & \text { if } \frac{\gamma_{1}}{g_{1}} \leq \xi, \ldots, \frac{\gamma_{N_{t}}}{g_{N_{t}}} \leq \xi, \\ \underset{k \in\left\{1, \ldots, N_{t}\right\}}{\arg \max }\left\{\frac{\gamma_{k}}{g_{k}}\right\}, & \text { else. }\end{cases}
$$

3) Difference Selection (DS) Rule [13]: Among the antennas $1, \ldots, N_{t}$, it selects the one that maximizes the weighted difference $\delta \gamma_{k}-(1-\delta) g_{k}$, where $\delta \in[0,1]$.

In order to ensure a fair comparison, the parameters $\delta$, $\beta$, and $\xi$ of the DS, EMI, and EMSLIR rules, respectively, are chosen to ensure that the interference-outage constraint of these rules is met with equality in their respective constrained regions.

Figs. 7a and 7b compare the average SEP of the optimal rule with the above TAS rules for full and partial CSI cases, respectively. (i) Full CSI: Here, in the constrained region $(\tau<16.5 \mathrm{~dB})$, the optimal rule outperforms all the other TAS rules. For example, at $\tau=14.5 \mathrm{~dB}$, its average SEP is lower by a factor of 13, 73, and 14 than the DS, EMI, and EMSLIR rules, respectively. Thus, there is a significant reduction in the average SEP. Equivalently, this results in a reduction in the transmit power $P_{t}$ required to achieve the same average SEP. In the unconstrained region $(\tau \geq 16.5 \mathrm{~dB})$, the DS (with $\delta=1$ ) and optimal rules reduce to the unconstrained rule in (8). Thus, their average SEPs saturate to the same value. The average SEPs of the EMI and EMSLIR rules also saturate but to much higher values. (ii) Partial CSI: Here, in the constrained region $(\tau<16.5 \mathrm{~dB})$ the trends are different.

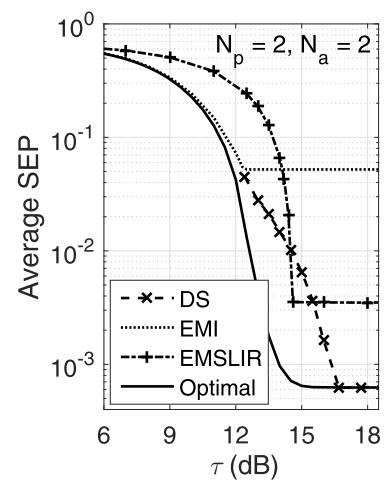

(a) Full CSI

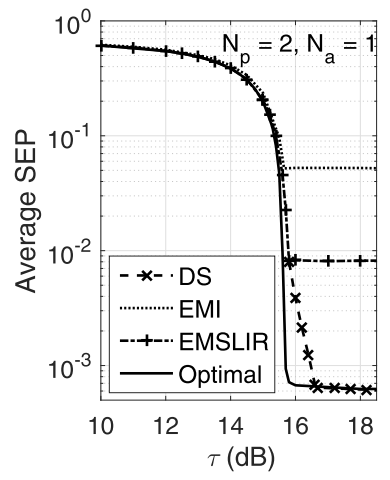

(b) Partial CSI
Fig. 7. Performance benchmarking: Average SEP as a function of $\tau$ for different TAS rules $\left(O_{\max }=0.1, N_{t}=4, N_{r}=1, P_{t}=12 \mathrm{~dB}, \kappa=1\right.$, and QPSK with $c_{1}=0.5$ and $c_{2}=0.6$ ).

We see that the EMI and EMSLIR rules are near-optimal for $\tau \leq 15 \mathrm{~dB}$. This happens due to an increase in the probability of $s=0$. However, for $\tau>15 \mathrm{~dB}$, these rules differ significantly. At $\tau=16 \mathrm{~dB}$, the average SEP of the optimal rule is lower by a factor of 6,83 , and 13 than the DS, EMI, and EMSLIR rules, respectively. In the unconstrained region $(\tau \geq 16.5 \mathrm{~dB})$ the behavior of the DS and optimal rules is the same as in the full CSI case. However, the average SEP of the EMSLIR rule saturates to a higher value than that for full CSI, while that of the EMI rule saturates to the same value. The DS rule curve only exists for $\tau \geq 12.4 \mathrm{~dB}$ for full CSI and $\tau \geq 16.0 \mathrm{~dB}$ for partial CSI since it cannot satisfy the interference-outage constraint for smaller $\tau$.

\section{CONClusions}

We derived an SEP-optimal TAS rule for an underlay CR system in which a secondary system co-existed with multiple PRxs and had partial CSI of its many links to them. We saw that for an interference-outage constraint that was designed for multiple PRxs, the optimal rule took a novel form that brought out the role of partial CSI and the interference constraint. For STx-PRx links whose instantaneous channel power gains were known to the STx, it checked if these were below a threshold. It then scaled this by a factor that was a function of the statistics of the channel gains that were not known to the STx and a penalization factor that depended on the parameters of the interference-outage constraint. We then derived general expressions and bounds for the interference-outage probability and average SEP. We saw that the trends were different for full and partial CSI in the interference-constrained region.

An interesting avenue for future work is to develop the jointly optimal power adaptation and antenna selection rule when the transmit power can be varied continuously. Also, the analysis when the channel gains are correlated is a challenging open problem.

\section{APPENDIX}

\section{A. Proof of Result 1}

In order to prove this result, we introduce the following terminology. We define a feasible rule to be a TAS rule that 
satisfies the interference-outage constraint in (6). Let $\mathcal{F}$ denote the set of all feasible rules. It is non-empty as the TAS rule that always selects antenna 0 has an interference-outage probability of zero, and is, therefore, feasible. Consider the following cases.

1. $O_{u} \leq O_{\max }$ : Here, the unconstrained rule is feasible. It clearly yields the lowest SEP.

2. $O_{u}>O_{\max }$ : Consider any feasible rule $\phi \in \mathcal{F}$. It selects antenna $s=\phi\left(\mathbf{H}, \mathbf{G}_{\mathcal{A}}\right)$. For a given $\lambda>0$, define

$\psi_{s}(\lambda) \triangleq \mathbb{E}_{\mathbf{H}, \mathbf{G}_{\mathcal{A}}}\left[\operatorname{SEP}\left(\gamma_{s}\right)\right]-\lambda \alpha_{s}\left(\mathcal{A}^{c}\right) \mathbb{E}_{\mathbf{H}, \mathbf{G}_{\mathcal{A}}}\left[I_{\left\{L_{s}(\mathcal{A})\right\}}\right]$.

From (11), recall that $\alpha_{s}\left(\mathcal{A}^{c}\right)=\operatorname{Pr}\left(D_{s}\left(\mathcal{A}^{c}\right)\right)$. Also, $\mathbb{E}_{\mathbf{H}, \mathbf{G}_{\mathcal{A}}}\left[I_{\left\{L_{s}(\mathcal{A})\right\}}\right]=\operatorname{Pr}\left(L_{s}(\mathcal{A})\right)$. Since $g_{1 s}, \ldots, g_{N_{p} s}$ are independent, it follows that

$$
\begin{aligned}
\operatorname{Pr}\left(D_{s}\left(\mathcal{A}^{c}\right)\right) \operatorname{Pr}\left(L_{s}(\mathcal{A})\right) & =\operatorname{Pr}\left(D_{s}\left(\mathcal{A}^{c}\right) \cap L_{s}(\mathcal{A})\right), \\
& =\operatorname{Pr}\left(\bigcap_{i=1}^{N_{p}}\left\{P_{t} g_{i s} \leq \tau\right\}\right) .
\end{aligned}
$$

Hence, $\psi_{s}(\lambda)=\mathbb{E}_{\mathbf{H}, \mathbf{G}_{\mathcal{A}}}\left[\operatorname{SEP}\left(\gamma_{s}\right)\right]-\lambda \operatorname{Pr}(\overbrace{i=1}^{N_{p}}\left\{P_{t} g_{i s} \leq \tau\right\})$. From the definition of $s^{*}$ in (12), it follows that $\psi_{s^{*}}(\lambda) \leq$ $\psi_{s}(\lambda)$. Therefore, for any $\phi \in \mathcal{F}$,

$$
\begin{aligned}
& \mathbb{E}_{\mathbf{H}, \mathbf{G}_{\mathcal{A}}}\left[\operatorname{SEP}\left(\gamma_{s^{*}}\right)\right]-\lambda \operatorname{Pr}\left(\underset{i=1}{N_{p}}\left\{P_{t} g_{i s^{*}} \leq \tau\right\}\right) \\
& \leq \mathbb{E}_{\mathbf{H}, \mathbf{G}_{\mathcal{A}}}\left[\operatorname{SEP}\left(\gamma_{s}\right)\right]-\lambda \operatorname{Pr}(\overbrace{i=1}^{N_{p}}\left\{P_{t} g_{i s} \leq \tau\right\}) .
\end{aligned}
$$

Now, chose $\lambda>0$ such that $\operatorname{Pr}\left(\underset{i=1}{N_{p}}\left\{P_{t} g_{i s^{*}} \leq \tau\right\}\right)=1-$ $O_{\max }$. That such a $\lambda$ exists is proved in Lemma 2 below. Thus, $\phi^{*}$ is also a feasible rule. Rearranging terms in (39), we get

$$
\begin{aligned}
& \mathbb{E}_{\mathbf{H}, \mathbf{G}_{\mathcal{A}}}\left[\operatorname{SEP}\left(\gamma_{s^{*}}\right)\right] \\
& \leq \mathbb{E}_{\mathbf{H}, \mathbf{G}_{\mathcal{A}}}\left[\operatorname{SEP}\left(\gamma_{s}\right)\right] \\
& -\lambda\left(\operatorname{Pr}\left(\bigcap_{i=1}^{N_{p}}\left\{P_{t} g_{i s} \leq \tau\right\}\right)-\left(1-O_{\max }\right)\right) .
\end{aligned}
$$

As $\lambda>0$ and $\phi$ is feasible, we get $\mathbb{E}_{\mathbf{H}, \mathbf{G}_{\mathcal{A}}}\left[\operatorname{SEP}\left(\gamma_{s^{*}}\right)\right] \leq$ $\mathbb{E}_{\mathbf{H}, \mathbf{G}_{\mathcal{A}}}\left[\operatorname{SEP}\left(\gamma_{s}\right)\right]$. Thus, $\phi^{*}$ is SEP-optimal.

Lemma 2: For any $O_{\max } \in\left[0, O_{u}\right)$, a $\lambda>0$ exists such that $O_{\lambda}$ is equal to $O_{\max }$.

Proof: The expression for the interference-outage probability of the selection rule in (12) is given in (15). This derivation is shown in Appendix VI-B to ensure flow and is not repeated here. From (15), we see that for a continuous fading model, $O_{\lambda}$ is a continuous function of $\lambda$. Furthermore, it is equal to $O_{u}$ when $\lambda=0$ and is 0 when $\lambda=c_{1} /(1-\alpha)$. Thus, by the intermediate value theorem, for every $0 \leq O_{\max }<O_{u}$, there exists a corresponding $\lambda \in\left(0, c_{1} /(1-\alpha)\right]$ at which $O_{\lambda}=O_{\max }$.

\section{B. Proof of Lemma 1}

1) Derivation of Exact Expression: We have

$$
O_{\lambda}=1-\operatorname{Pr}\left(B_{s}\right),
$$

where $B_{s} \triangleq \cap_{i=1}^{N_{p}}\left\{P_{t} g_{i s} \leq \tau\right\}$. By the law of total probability,

$$
O_{\lambda}=1-\operatorname{Pr}\left(s=0, B_{0}\right)-\sum_{k=1}^{N_{t}} \operatorname{Pr}\left(s=k, B_{k}\right) .
$$

Since the statistics of $h_{1 k}, \ldots, h_{N_{t} k}$ and $g_{1 k}, \ldots, g_{N_{p} k}$ are the same for all $k \in\left\{1,2, \ldots, N_{t}\right\}$, and $g_{10}=0, \ldots, g_{N_{p} 0}=0$, it follows that

$$
O_{\lambda}=1-\operatorname{Pr}(s=0)-N_{t} \operatorname{Pr}\left(s=1, B_{1}\right) .
$$

We evaluate the two probability terms in (43) separately below.

a) Expression for $\operatorname{Pr}(s=0)$ : From (12), we have

$$
\begin{aligned}
& \operatorname{Pr}(s=0)=\operatorname{Pr}\left(y_{1}-\lambda \alpha I_{\left\{L_{1}\right\}}\right.>c_{1}-\lambda, \ldots, \\
&\left.y_{N_{t}}-\lambda \alpha I_{\left\{L_{N_{t}}\right\}}>c_{1}-\lambda\right) .
\end{aligned}
$$

Using the fact that the STx-SRx and STx-PRx channel gains of different antennas are independent, we get

$$
\operatorname{Pr}(s=0)=\prod_{k=1}^{N_{t}} \operatorname{Pr}\left(y_{k}-\lambda \alpha I_{\left\{L_{k}\right\}}>c_{1}-\lambda\right) .
$$

Using the law of total probability,

$$
\begin{aligned}
& \operatorname{Pr}\left(y_{k}-\lambda \alpha I_{\left\{L_{k}\right\}}>c_{1}-\lambda\right) \\
& \quad=p_{l} \operatorname{Pr}\left(y_{k}-\lambda \alpha I_{\left\{L_{k}\right\}}>c_{1}-\lambda \mid L_{k}\right) \\
& \quad+\left(1-p_{l}\right) \operatorname{Pr}\left(y_{k}-\lambda \alpha I_{\left\{L_{k}\right\}}>c_{1}-\lambda \mid L_{k}^{c}\right),
\end{aligned}
$$

where $p_{l}=\operatorname{Pr}\left(L_{k}\right)$ is given in (14). Given $L_{k}$, i.e., $I_{\left\{L_{k}\right\}}=1$, we have $y_{k}-\lambda \alpha I_{\left\{L_{k}\right\}}=y_{k}-\lambda \alpha$. Similarly, given $L_{k}^{c}$, we have $y_{k}-\lambda \alpha I_{\left\{L_{k}\right\}}=y_{k}$. Hence,

$$
\begin{aligned}
\operatorname{Pr}\left(y_{k}-\lambda \alpha I_{\left\{L_{k}\right\}}>c_{1}-\lambda\right)= & p_{l} \operatorname{Pr}\left(y_{k}>c_{1}-\lambda(1-\alpha)\right) \\
& +\left(1-p_{l}\right) \operatorname{Pr}\left(y_{k}>c_{1}-\lambda\right) .
\end{aligned}
$$

Writing (47) in terms of the CCDF of the RV $y_{k}$ and then substituting it in (45), we get

$$
\operatorname{Pr}(s=0)=\Psi_{0},
$$

where $\Psi_{0}$ is defined in the lemma statement.

b) Expression for $\operatorname{Pr}\left(s=1, B_{1}\right)$ : Let $n_{c}$ antennas among the antennas $2, \ldots, N_{t}$ be $\mathcal{A}$-outage-compatible. Such $n_{c}$ antennas can be chosen in $\left(\begin{array}{c}N_{t}-1 \\ n_{c}\end{array}\right)$ ways. One such combination is that antennas $2, \ldots, n_{c}+1$ are $\mathcal{A}$-outage-compatible and antennas $n_{c}+2, \ldots, N_{t}$ are not. This event can be written as $L_{2} \cap \ldots \cap L_{n_{c}+1} \cap L_{n_{c}+2}^{c} \cap \ldots \cap L_{N_{t}}^{c}$. By symmetry and the law of total probability, we get

$$
\operatorname{Pr}\left(s=1, B_{1}\right)=\sum_{n_{c}=0}^{N_{t}-1}\left(\begin{array}{c}
N_{t}-1 \\
n_{c}
\end{array}\right) \operatorname{Pr}(s=1 \mid E) \operatorname{Pr}(E),
$$

where $E \triangleq B_{1} \cap L_{2} \cap \ldots \cap L_{n_{c}+1} \cap L_{n_{c}+2}^{c} \cap \ldots \cap L_{N_{t}}^{c}$. Since the STx-PRx channel gains are independent, it can be seen that $\operatorname{Pr}(E)=\operatorname{Pr}\left(B_{1}\right) \operatorname{Pr}\left(L_{2}\right) \ldots \operatorname{Pr}\left(L_{n_{c}+1}\right)$ $\times \operatorname{Pr}\left(L_{n_{c}+2}^{c}\right) \ldots \operatorname{Pr}\left(L_{N_{t}}^{c}\right)$. From (10) and (14), we get

$$
\operatorname{Pr}(E)=\left(1-O_{u}\right) p_{l}^{n_{c}}\left(1-p_{l}\right)^{N_{t}-n_{c}-1} .
$$


To obtain $\operatorname{Pr}(s=1 \mid E)$, we first condition over $y_{1}=x$ and derive an expression for $\operatorname{Pr}\left(s=1 \mid E, y_{1}=x\right)$. Given $E$ and $y_{1}=x$, the optimal rule selects antenna 1 if $x-\lambda \alpha<c_{1}-\lambda$, $x-\lambda \alpha<y_{m}-\lambda \alpha$, for $2 \leq m \leq n_{c}+1$, and $x-\lambda \alpha<y_{j}$, for $n_{c}+2 \leq j \leq N_{t}$. Thus,

$$
\begin{aligned}
\operatorname{Pr}(s= & \left.1 \mid E, y_{1}=x\right) \\
= & \operatorname{Pr}\left(x-\lambda \alpha<c_{1}-\lambda,\right. \\
& x-\lambda \alpha<y_{2}-\lambda \alpha, \ldots, x-\lambda \alpha<y_{n_{c}+1}-\lambda \alpha, \\
& \left.x-\lambda \alpha<y_{n_{c}+2}, \ldots, x-\lambda \alpha<y_{N_{t}} \mid E, y_{1}=x\right) .
\end{aligned}
$$

Using the fact that $y_{2}, \ldots, y_{N_{t}}$ are i.i.d., we get

$$
\begin{aligned}
\operatorname{Pr}\left(s=1 \mid E, y_{1}=x\right)=I_{\left\{x<c_{1}-\lambda(1-\alpha)\right\}}[ & \left.\operatorname{Pr}\left(y_{2}>x\right)\right]^{n_{c}} \\
& \times\left[\operatorname{Pr}\left(y_{n_{c}+2}>x-\lambda \alpha\right)\right]^{N_{t}-n_{c}-1} .
\end{aligned}
$$

Averaging over $y_{1}$ and writing in terms of its CCDF and PDF, we get

$$
\begin{aligned}
\operatorname{Pr}(s=1 \mid E)= & \int_{0}^{c_{1}-\lambda(1-\alpha)}\left[F_{y}^{c}(x)\right]^{n_{c}} \\
& \times\left[F_{y}^{c}(x-\lambda \alpha)\right]^{N_{t}-n_{c}-1} f_{y}(x) d x .
\end{aligned}
$$

Substituting (53) and (50) in (49) yields the expression for $\operatorname{Pr}\left(s=1, B_{1}\right)$. Finally, substituting this along with (48) in (43) yields (15).

2) Derivation of Bound: We split the region of integration in (15) into two parts $I_{1}$ and $I_{2}$, where we integrate over $(0, \lambda \alpha]$ in $I_{1}$ and over $\left(\lambda \alpha, c_{1}-\lambda(1-\alpha)\right]$ in $I_{2}$. Using the fact that $F_{y}^{c}(x-\lambda \alpha)=1$, for $x \leq \lambda \alpha$, we get

$$
\begin{aligned}
I_{1} & =\int_{0}^{\lambda \alpha}\left[1-p_{l}+p_{l} F_{y}^{c}(x)\right]^{N_{t}-1} f_{y}(x) d x, \\
& =\frac{1}{N_{t} p_{l}}\left(1-\left[1-p_{l}+p_{l} F_{y}^{c}(\lambda \alpha)\right]^{N_{t}}\right),
\end{aligned}
$$

and

$$
\begin{aligned}
I_{2}=\int_{\lambda \alpha}^{c_{1}-\lambda(1-\alpha)}\left[\left(1-p_{l}\right) F_{y}^{c}(x-\lambda \alpha)+\right. & \left.p_{l} F_{y}^{c}(x)\right]^{N_{t}-1} \\
& \times f_{y}(x) d x .
\end{aligned}
$$

For $x \leq c_{1}-\lambda(1-\alpha), F_{y}^{c}(x-\lambda \alpha) \geq F_{y}^{c}\left(c_{1}-\lambda\right)$. Substituting this in (56) yields

$$
I_{2} \geq \frac{1}{N_{t} p_{l}}\left(\left[\left(1-p_{l}\right) F_{y}^{c}\left(c_{1}-\lambda\right)+p_{l} F_{y}^{c}(\lambda \alpha)\right]^{N_{t}}-\Psi_{0}\right) .
$$

Combining (54) and (57) and substituting it in (15) yields (16).

\section{Brief Proof of Result 2}

We show the key steps and skip some involved calculations. The average SEP can be written as $\overline{\mathrm{SEP}}=\mathbb{E}_{\mathbf{y}}[\operatorname{Pr}(\operatorname{Err} \mid \mathbf{y})]$, where $\mathbf{y} \triangleq\left[y_{1}, \ldots, y_{N_{t}}\right]$ and Err is the decoding error event. Using the law of total probability, we can write

$$
\operatorname{Pr}(\operatorname{Err} \mid \mathbf{y})=\operatorname{Pr}(s=0, \operatorname{Err} \mid \mathbf{y})+\sum_{k=1}^{N_{t}} \operatorname{Pr}(s=k, \operatorname{Err} \mid \mathbf{y}) .
$$

Furthermore, using chain rule, we get $\operatorname{Pr}(s=k, \operatorname{Err} \mid \mathbf{y})=$ $\operatorname{Pr}(s=k \mid \mathbf{y}) \operatorname{Pr}(\operatorname{Err} \mid \mathbf{y}, s=k)$, for $0 \leq k \leq N_{t}$. By exploiting symmetry, $\overline{\mathrm{SEP}}$ can be written as

$$
\begin{aligned}
\overline{\mathrm{SEP}}=\mathbb{E}_{\mathbf{y}}[\operatorname{Pr}(s=0 \mid \mathbf{y}) \operatorname{Pr}(\operatorname{Err} \mid \mathbf{y}, s=0)] \\
+N_{t} \mathbb{E}_{\mathbf{y}}[\operatorname{Pr}(s=1 \mid \mathbf{y}) \operatorname{Pr}(\operatorname{Err} \mid \mathbf{y}, s=1)] .
\end{aligned}
$$

Given $s=0$, the SEP is equal to ${ }^{2} e_{0}=1-(1 / M)$, and given $s=1$, it is equal to $y_{1}$. Hence,

$$
\overline{\mathrm{SEP}}=e_{0} \mathbb{E}_{\mathbf{y}}[\operatorname{Pr}(s=0 \mid \mathbf{y})]+N_{t} \mathbb{E}_{\mathbf{y}}\left[y_{1} \operatorname{Pr}(s=1 \mid \mathbf{y})\right] .
$$

From the law of total expectation, we know that $\mathbb{E}_{\mathbf{y}}[\operatorname{Pr}(s=0 \mid \mathbf{y})]=\operatorname{Pr}(s=0)$. Similarly,

$$
\mathbb{E}_{\mathbf{y}}\left[y_{1} \operatorname{Pr}(s=1 \mid \mathbf{y})\right]=\mathbb{E}_{y_{1}}\left[y_{1} \operatorname{Pr}\left(s=1 \mid y_{1}\right)\right] .
$$

Substituting these two results into (60), we get $\overline{\mathrm{SEP}}=T_{1}+$ $N_{t} \mathbb{E}_{y_{1}}\left[y_{1} \operatorname{Pr}\left(s=1 \mid y_{1}\right)\right]$, where $T_{1}=e_{0} \operatorname{Pr}(s=0)$. Substituting (48) yields the expression for $T_{1}$ in (26).

Using the law of total probability, we get

$$
N_{t} \mathbb{E}_{y_{1}}\left[y_{1} \operatorname{Pr}\left(s=1 \mid y_{1}\right)\right]=T_{2}+T_{3},
$$

where $T_{2}=N_{t} \mathbb{E}_{y_{1}}\left[y_{1} \operatorname{Pr}\left(s=1, L_{1} \mid y_{1}\right)\right]$ and $T_{3}=$ $N_{t} \mathbb{E}_{y_{1}}\left[y_{1} \operatorname{Pr}\left(s=1, L_{1}^{c} \mid y_{1}\right)\right]$.

Expression for $\operatorname{Pr}\left(s=1, L_{1} \mid y_{1}\right)$ : Along lines similar to (49), which fixed the number of antennas that are $\mathcal{A}$-outagecompatible, we can show that

$$
\begin{aligned}
\operatorname{Pr}(s= & \left.1, L_{1} \mid y_{1}\right) \\
& =\sum_{n_{c}=0}^{N_{t}-1}\left(\begin{array}{c}
N_{t}-1 \\
n_{c}
\end{array}\right) \operatorname{Pr}\left(s=1 \mid B, y_{1}\right) \operatorname{Pr}\left(B \mid y_{1}\right),
\end{aligned}
$$

where $B=L_{1} \cap L_{2} \cap \ldots \cap L_{n_{c}+1} \cap L_{n_{c}+2}^{c} \cap \ldots \cap L_{N_{t}}^{c}$. As the STx-PRx channels are mutually independent and are also independent of $y_{1}$, using the definition of $p_{l}$ in (14), we get

$$
\operatorname{Pr}\left(B \mid y_{1}\right)=\operatorname{Pr}(B)=p_{l}^{n_{c}+1}\left(1-p_{l}\right)^{N_{t}-n_{c}-1} .
$$

Simplifying along lines similar to those from (51) to (52),

$$
\begin{aligned}
& \operatorname{Pr}\left(s=1 \mid B, y_{1}\right)=I_{\left\{y_{1}<c_{1}-\lambda(1-\alpha)\right\}}\left[F_{y}^{c}\left(y_{1}\right)\right]^{n_{c}} \\
& \times\left[F_{y}^{c}\left(y_{1}-\lambda \alpha\right)\right]^{N_{t}-n_{c}-1} .
\end{aligned}
$$

Substituting (65) and the expression for $\operatorname{Pr}\left(B \mid y_{1}\right)$ from (64) in $(63)$ yields

$$
\begin{aligned}
\operatorname{Pr}\left(s=1, L_{1} \mid y_{1}\right)=\left[\left(1-p_{l}\right) F_{y}^{c}\right. & \left.\left(y_{1}-\lambda \alpha\right)+p_{l} F_{y}^{c}\left(y_{1}\right)\right]^{N_{t}-1} \\
& \times p_{l} I_{\left\{y_{1}<c_{1}-\lambda(1-\alpha)\right\}}
\end{aligned}
$$

Substituting (66) in $N_{t} \mathbb{E}_{y_{1}}\left[y_{1} \operatorname{Pr}\left(s=1, L_{1} \mid y_{1}\right)\right]$ and averaging over $y_{1}$ yields $T_{2}$ in (27).

Similarly, $\operatorname{Pr}\left(s=1, L_{1}^{c} \mid y_{1}\right)$ can be shown to simplify to

$$
\begin{array}{r}
\operatorname{Pr}\left(s=1, L_{1}^{c} \mid y_{1}\right)=\left[\left(1-p_{l}\right) F_{y}^{c}\left(y_{1}\right)+p_{l} F_{y}^{c}\left(y_{1}+\lambda \alpha\right)\right]^{N_{t}-1} \\
\times\left(1-p_{l}\right) I_{\left\{y_{1}<c_{1}-\lambda\right\}}
\end{array}
$$

Substituting (67) in $N_{t} \mathbb{E}_{y_{1}}\left[y_{1} \operatorname{Pr}\left(s=1, L_{1}^{c} \mid y_{1}\right)\right]$ and averaging over $y_{1}$ yields $T_{3}$ in (28).

\footnotetext{
${ }^{2}$ To ensure an accurate analysis, we use the exact value of the SEP for $s=0$ in this derivation and not its approximate value of $c_{1}$, which was used in Section II-C.
} 


\section{REFERENCES}

[1] M. Sherman, A. N. Mody, R. Martinez, C. Rodriguez, and R. Reddy, "IEEE standards supporting cognitive radio and networks, dynamic spectrum access, and coexistence," IEEE Commun. Mag., vol. 46, no. 7, pp. 72-79, Jul. 2008.

[2] A. Goldsmith, S. A. Jafar, I. Maric, and S. Srinivasa, "Breaking spectrum gridlock with cognitive radios: An information theoretic perspective," Proc. IEEE, vol. 97, no. 5, pp. 894-914, Apr. 2009.

[3] P. L. Yeoh, M. Elkashlan, T. Q. Duong, N. Yang, and D. B. da Costa, "Transmit antenna selection for interference management in cognitive relay networks," IEEE Trans. Veh. Technol., vol. 63, no. 7, pp. 3250-3262, Sep. 2014.

[4] P. L. Yeoh, M. Elkashlan, K. J. Kim, T. Q. Duong, and G. K. Karagiannidis, "Cognitive MIMO relaying with multiple primary transceivers," in Proc. IEEE Globecom, Dec. 2013, pp. 1956-1961.

[5] V.-D. Nguyen, C. T. Nguyen, H. V. Nguyen, and O.-S. Shin, "Joint beamforming and antenna selection for sum rate maximization in cognitive radio networks," IEEE Commun. Lett., vol. 21, no. 6, pp. 1369-1372, Jun. 2017

[6] M. Hanif, H.-C. Yang, and M.-S. Alouini, "Transmit antenna selection for underlay cognitive radio with instantaneous interference constraint," in Proc. IEEE Globecom, Dec. 2015, pp. 1-5.

[7] H. Wang, J. Lee, S. Kim, and D. Hong, "Capacity enhancement of secondary links through spatial diversity in spectrum sharing," IEEE Trans. Wireless Commun., vol. 9, no. 2, pp. 494-499, Feb. 2010.

[8] S. Kashyap and N. B. Mehta, "Optimal binary power control for underlay CR with different interference constraints and impact of channel estimation errors," IEEE Trans. Commun., vol. 62, no. 11, pp. 3753-3764, Nov. 2014.

[9] L. Sboui, Z. Rezki, and M. S. Alouini, "A unified framework for the ergodic capacity of spectrum sharing cognitive radio systems," IEEE Trans. Wireless Commun., vol. 12, no. 2, pp. 877-887, Feb. 2013.

[10] R. Sarvendranath and N. B. Mehta, "Transmit antenna selection for interference-outage constrained underlay CR," IEEE Trans. Commun., vol. 66, no. 9, pp. 3772-3783, Sep. 2018.

111] R. Sarvendranath and N. B. Mehta, "Antenna selection in interferenceconstrained underlay cognitive radios: SEP-optimal rule and performance benchmarking," IEEE Trans. Commun., vol. 61, no. 2, pp. 496-506, Feb. 2013.

[12] R. Sarvendranath and N. B. Mehta, "Antenna selection with power adaptation in interference-constrained cognitive radios," IEEE Trans. Commun., vol. 62, no. 3, pp. 786-796, Mar. 2014.

[13] Y. Wang and J. P. Coon, "Difference antenna selection and power allocation for wireless cognitive systems," IEEE Trans. Commun., vol. 59, no. 12, pp. 3494-3503, Dec. 2011.

[14] R. Zhang and Y.-C. Liang, "Exploiting multi-antennas for opportunistic spectrum sharing in cognitive radio networks," IEEE J. Sel. Topics Signal Process., vol. 2, no. 1, pp. 88-102, Feb. 2008.

[15] G. Scutari, D. P. Palomar, and S. Barbarossa, "Cognitive MIMO radio," IEEE Signal Process. Mag., vol. 25, no. 6, pp. 46-59, Nov. 2008.

[16] A. F. Molisch and M. Z. Win, "MIMO systems with antenna selection," IEEE Commun. Mag., vol. 5, no. 1, pp. 46-56, Mar. 2004.

[17] Evolved Universal Terrestrial Radio Access (E-UTRA); Physical Channels and Modulation (Release 8), document 36.211 (v8.4.0), 3rd Generation Partnership Project, 2008.

[18] N. B. Mehta, S. Kashyap, and A. F. Molisch, "Antenna selection in LTE: From motivation to specification," IEEE Commun. Mag., vol. 50, no. 10, pp. 144-150, Oct. 2012.

[19] J. Zhou and J. Thompson, "Single-antenna selection for MISO cognitive radio," in Proc. IET Seminar Cogn. Radio Softw. Defined Radios, Sep. 2008, pp. 1-5.

[20] R. Zhang, F. Gao, and Y.-C. Liang, "Cognitive beamforming made practical: Effective interference channel and learning-throughput tradeoff," IEEE Trans. Commun., vol. 58, no. 2, pp. 706-718, Feb. 2010.

[21] L. Zhang et al., "Primary channel gain estimation for spectrum sharing in cognitive radio networks," IEEE Trans. Commun., vol. 65, no. 10, pp. 4152-4162, Oct. 2017.
[22] R. Zhang, "On active learning and supervised transmission of spectrum sharing based cognitive radios by exploiting hidden primary radio feedback," IEEE Trans. Commun., vol. 58, no. 10, pp. 2960-2970, Oct. 2010.

[23] L. Musavian and S. Aissa, "Fundamental capacity limits of cognitive radio in fading environments with imperfect channel information," IEEE Trans. Commun., vol. 57, no. 11, pp. 3472-3480, Nov. 2009.

[24] H. A. Suraweera, P. J. Smith, and M. Shafi, "Capacity limits and performance analysis of cognitive radio with imperfect channel knowledge," IEEE Trans. Veh. Technol., vol. 59, no. 4, pp. 1811-1822, May 2010.

[25] F. A. Khan, K. Tourki, M.-S. Alouini, and K. A. Qaraqe, "Performance analysis of a power limited spectrum sharing system with TAS/MRC," IEEE Trans. Signal Process., vol. 62, no. 4, pp. 954-967, Feb. 2014.

[26] A. J. Goldsmith, Wireless Communication. Cambridge, U.K.: Cambridge Univ. Press, 2005.

[27] S. T. Chung and A. J. Goldsmith, "Degrees of freedom in adaptive modulation: A unified view," IEEE Trans. Commun., vol. 49, no. 9, pp. 1561-1571, Sep. 2001

[28] M. Abramowitz and I. A. Stegun, Eds., Handbook of Mathematical Functions: With Formulas, Graphs, and Mathematical Tables, 9th ed. New York, NY, USA: Dover, 1972

[29] L. S. Gradshteyn and L. M. Ryzhik, Table of Integrals, Series, and Products. New York, NY, USA: Academic, 2000.

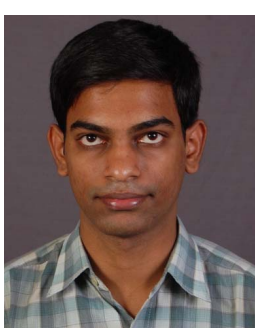

Rimalapudi Sarvendranath (S'12) received the B.Tech. degree in electrical and electronics engineering from the National Institute of Technology Karnataka, Surathkal, in 2009, and the M.E. degree from the Department of Electrical Communication Engineering, Indian Institute of Science (IISc), Bengaluru, India, in 2012, where he is currently pursuing the Ph.D. degree with the ECE Department. From 2009 to 2010, he was a Research Assistant with the Department of Instrumentation, IISc, where he was involved in the development of image processing algorithms. From 2012 to 2016, he was with Broadcom Communications Technologies Pvt. Ltd., Bengaluru, where he worked on the development and implementation of algorithms for LTE and IEEE 802.11ac wireless standards. His research interests include wireless communication, multiple antenna techniques, cognitive radio, and next generation wireless standards.

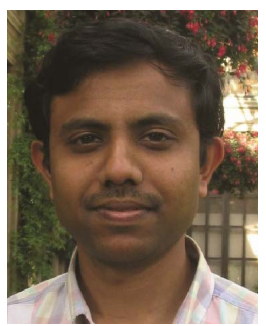

Neelesh B. Mehta (S'98-M'01-SM'06-F'19) received the B.Tech. degree in electronics and communications engineering from IIT Madras in 1996, and the M.S. and Ph.D. degrees in electrical engineering from the California Institute of Technology, Pasadena, CA, USA, in 1997 and 2001, respectively. $\mathrm{He}$ is currently a Professor with the Department of Electrical Communication Engineering, Indian Institute of Science, Bengaluru. He is a Fellow of the IEEE, Indian National Academy of Engineering, and the National Academy of Sciences India. He was a recipient of the Shanti Swarup Bhatnagar Award in 2017 and the Swarnjayanti Fellowship. He served on the Board of Governors of IEEE ComSoc from 2012 to 2015. He served on the Executive Editorial Committee of the IEEE TRANSACTIONS ON WIRELESS COMMUNICATIONS from 2014 to 2017, and as its Chair from 2017 to 2018. He is currently an Editor of the IEEE TRANSACTIONS ON COMMUNICATIONS. 